\title{
Interférences
}

Ars scribendi

\section{La part biographique dans une œuvre historique condensée}

L'exemple de Florus

\section{Guillaume Flamerie de Lachapelle}

\section{(2) OpenEdition}

\section{Journals}

Édition électronique

URL : http://journals.openedition.org/interferences/913

DOI : 10.4000/interferences.913

ISSN : $1777-5485$

\section{Éditeur}

HiSoMA - Histoire et sources des Mondes antiques

Édition imprimée

Date de publication : 1 janvier 2009

ISSN : 1777-5485

\section{Référence électronique}

Guillaume Flamerie de Lachapelle, «La part biographique dans une œuvre historique condensée », Interférences [En ligne], 5 | 2009, mis en ligne le 10 juillet 2014, consulté le 15 septembre 2020. URL : http://journals.openedition.org/interferences/913 ; DOI : https://doi.org/10.4000/interferences.913

Ce document a été généré automatiquement le 15 septembre 2020.

Tous droits réservés 


\title{
La part biographique dans une œuvre historique condensée
}

\author{
L'exemple de Florus
}

Guillaume Flamerie de Lachapelle

1 Le moins qu'on puisse dire de l'œuvre de Florus ${ }^{1}$ est qu'elle ne jouit pas d'une grande faveur auprès de la plupart des historiens. On ne prête en effet quelque attention à notre auteur que dans le cadre d'une Quellenforschung dans laquelle il est seulement un maillon sans intérêt intrinsèque, ou pour examiner des événements dont il est l'unique garant. Aux yeux de la science positiviste, l'Epitome s'est longtemps réduite à un amas de figures vulgaires et de mauvais goût, surtout remarquable par la série d'anachronismes, d'erreurs flagrantes ou de mensonges qu'elle contient ${ }^{2}$. Se pencher sur un tel écrivain peut donc sembler singulier: se pencher sur un tel écrivain pour parler de biographie paraitra même, de prime abord, tout à fait absurde.

2 On a souvent noté en effet que des personnages centraux de l'histoire romaine, comme Hamilcar Barca lors de la première guerre punique, ou L. Catulus au cours de la guerre contre les Cimbres, ne sont pas même cités ${ }^{3}$, et l'on pourrait d'ailleurs s'étonner qu'une telle œuvre ait été écrite à un moment où le genre biographique s'épanouit dans la littérature latine ${ }^{4}$. En fait, le sujet de la biographie ne parait pertinent que si l'on considère que Florus écrit la vie du populus Romanus : on pourrait parler dans ce cas, à la manière d'un critique récent, d'une «römische Geschichte in einer biographischen Form ${ }^{5}$ ", à travers une éventuelle influence de la méthode suétonienne ${ }^{6}$. Mais de biographies individuelles, l'Epitome ne contient apparemment aucune trace.

3 La question que nous nous poserons sera donc la suivante: sur un terrain hostile comme l'Epitome, quelle place peut se frayer la biographie d'agents individuels?

4 Pour apporter une réponse, nous réfléchirons autour de trois obstacles, qui restreignent, dès le départ, la place du biographique dans le genre historiographique de l'abrégé en général, et de l'Epitome de Florus en particulier ${ }^{7}$. Nous verrons comment l'écrivain injecte tout de même à chaque fois, dans une part certes restreinte, mais réelle, un élément biographique. Les trois contraintes que nous analyserons 
successivement sont, bien sûr, étroitement liées entre elles, mais nous les distinguons ici, de façon quelque peu artificielle, afin de donner plus de clarté à notre exposé :

- Le plan stylistique est celui qui retient le plus immédiatement l'attention: la breuitas constamment recherchée par Florus parait incompatible avec les notices, les longues analyses et les digressions qui constituent souvent, dans un récit historique, les lieux où peut être dépeint tel ou tel héros.

- Le point de vue compositionnel joue aussi un rôle: la coexistence de méthodes chronologique et thématique étouffe à deux titres le développement d'une écriture biographique.

- Enfin, l'idéologie ou, pour mieux dire, la Weltanschauung qui sous-tend l'ensemble de l' Epitome s'oppose à ce que les individus émergent nettement.

\section{Obstacle stylistique : le goût de la breuitas}

\section{La breuitas dans le récit et dans la caractérisation des personnages}

5 La première contrainte, d'ordre stylistique, semble rédhibitoire: écrivant probablement à une époque où le goût littéraire se porte vers les compendia et les abrégés, où la pointe est plus que tout appréciée, et étant sans doute lui-même auteur d'épigrammes ${ }^{8}$, Florus a clairement choisi la brièveté ${ }^{9}$. C'est au pas de course en effet qu'il passe en revue huit siècles d'histoire romaine (de la fondation de Rome, en 753 av. J.-C., à la défaite de Varus, en 9 apr. J.-C.). Les héros succèdent aux scélérats, mais peu restent longtemps sur scène. Or le genre biographique suppose de se concentrer de façon prolongée sur un individu ${ }^{10}$. Si Florus avait choisi de consacrer plus d'espace à la narration des événements dont il s'occupe, il aurait pu, même dans une œuvre historiographique, accorder une place consistante à la biographie, en mettant en valeur par exemple, comme un Diodore de Sicile ou un Dion Cassius, une figure saillante au centre de tout un livre, de tout un récit, figure autour de laquelle s'organiseraient les événements ${ }^{11}$.

Rien de tel chez notre auteur. Pressés par le cours du temps, les personnages se résument généralement à leurs seules actions en rapport avec le reste du récit, ce qui est le signe d'une écriture de type purement historiographique ${ }^{12}:$ un début in medias res, supprimant plusieurs éléments biographiques, confère de la puissance et de la nervosité à l'écriture de Florus ${ }^{13}$, mais laisse dans l'ombre les hommes qui font l'histoire. De la même façon, les évaluations psychologiques et/ou morales qui caractérisent l'écriture biographique dans l'Antiquité ${ }^{14}$ sont souvent absentes de l' Epitome ${ }^{15}$.

7 En voici un exemple flagrant:

Metellus deinde totam insulam igni ferroque populatus intra castella et urbes redegit, Cnoson, Eleythernan et, ut Graeci dicere solent, urbium matrem, Cydoneam; adeoque saeue in captiuos consulebatur, ut ueneno se plerique conficerent, alii deditionem suam ad Pompeium absentem mitterent

Métellus ravagea ensuite l'île tout entière par le fer et par le feu, et en refoula les habitants à l'intérieur de ses forteresses et de ses villes, Cnossos, Elytherna et Cydonea, la mère des villes, comme disent les Grecs. Et il traitait si cruellement les prisonniers que la plupart s'achevèrent en s'empoisonnant, tandis que d'autres faisaient parvenir leur reddition à Pompée absent ${ }^{16}$. 
Dans ces lignes, Florus adopte une écriture annalistique qui laisse délibérément de côté les aspects biographiques et l'enrichissement des personnages (présentation des causes et des conséquences) au profit des seuls faits ${ }^{17}$. Ce mode de narration ne livre aucune information au lecteur sur l'origine de Métellus, ses qualités ou les conséquences que sa conduite en Crète auront pour lui-même.

9 Très souvent d'ailleurs, Florus écrase encore la part biographique en énumérant à la suite les actions de deux ou trois personnages, les liant entre eux et réduisant notablement leur dimension individuelle. Ce procédé concerne surtout les Romains, qui sont fréquemment relégués à la fin d'un chapitre, une fois présentée la guerre dans son ensemble ${ }^{18}$. Après avoir évoqué les périls que les Thraces faisaient courir à Rome, voici comment Florus résume l'action des généraux romains chargés de repousser le danger :

Didius uagos et libera populatione diffusos intra suam reppulit Thraciam. Drusus ulterius egit et uetuit transire Danuuium. Minucius toto uastauit Hebro, multis quidem amissis, dum per perfidum glacie flumen equitatur. Volso Rhodopen Caucasumque penetrauit. Curio Dacia tenus uenit, sed tenebras saltuum expauit. Appius in Sarmatas usque peruenit, Lucullus ad terminum gentium Tanain lacumque Maeotin

Alors que <les Thraces> erraient et, dispersés de tous côtés, se livraient librement au pillage, Didius les refoula dans leur Thrace. Drusus les repoussa encore plus loin et les empêcha de franchir le Danube. Minucius ravagea toute la région de l'Hèbre, non sans subir, il est vrai, de nombreuses pertes parmi sa cavalerie qui avançait sur la glace perfide du fleuve. Volson pénétra dans le Rhodope et le Caucase. Curion vint jusqu'en Dacie, mais fut effrayé par les ténèbres des forêts. Appius parvint jusque chez les Sarmates, Lucullus, jusqu'au bout du monde, au Tanais et au lac Méotis ${ }^{19}$.

Les magistrats ne sont plus que des instruments aux mains d'un écrivain avant tout désireux d'une concision frappante et spectaculaire. On aurait au demeurant tort de croire que l'historien réserve un tel traitement aux seconds couteaux: même Sylla, pendant la guerre sociale, se voit traité de la sorte ${ }^{20}$.

11 La breuitas qui imprègne le style de Florus prend encore d'autres formes si l'on considère la caractérisation des personnages proprement dite. Des figures complexes se réduisent ainsi à un seul aspect : perfidia ou saeuitia, pour des hostes; uirtus ou fortitudo, pour des Romains ${ }^{21}$.

12 Un tableau saisissant tient alors lieu à la fois de récit et d'analyse morale, comme à propos d'Antoine et Cléopâtre, dans cette phrase admirablement ciselée :

Hinc mulier Aegyptia ab ebrio imperatore pretium libidinum Romanum imperium petit

Puis une Égyptienne demanda à un général ivre l'Empire romain en paiement de ses faveurs ${ }^{22}$.

\section{Deux façons de composer avec la breuitas}

13 Florus réussit tout de même à composer avec ce cadre contraignant en utilisant deux ressources.

\section{Les biographies miniatures}

Maîtrisant parfaitement l'art de la pointe et de la concision, Florus écrit des sortes de biographies en modèle réduit, au moyen de divers procédés comme la concaténation, qui permet de résumer brièvement les étapes d'une vie : 
Ceterum Lusitanos Viriatus erexit, uir calliditatis acerrimae, qui ex uenatore latro, ex latrone subito dux atque imperator et, si fortuna cessisset, Hispaniae Romulus

Quant aux Lusitaniens, ils furent soulevés par Viriathe, homme d'une habileté consommée, de chasseur devenu brigand, tout à coup, chef et général et, si la chance l'avait servi, le Romulus de l'Hispanie ${ }^{23}$.

Cette évocation de la carrière de Viriathe n'était nullement nécessaire pour le projet d'ensemble de l'historien ${ }^{24}$ : si cet intérêt pour le chef hispanique peut s'expliquer par le patriotisme de Florus, chez qui on a parfois vu un Hispanique ${ }^{25}$, il nous parait plus vraisemblable d'y lire une sorte de pied de nez de la part du malicieux épigrammatiste : il aurait fort bien pu se livrer au genre biographique, mais n'en éprouve pas le besoin. Du reste, le même procédé de concaténation est employé à propos de Spartacus, preuve que Viriathe ne retient pas l'attention de Florus pour des raisons seulement sentimentales ${ }^{26}$.

\section{La disproportion}

Dans le début de sa Vie d'Alexandre, Plutarque précise qu'une des caractéristiques du genre biographique est la part belle faite aux anecdotes ${ }^{27}$. C'est ce parti que semble avoir souvent adopté Florus : peu soucieux d'exhaustivité, il n'hésite pas, pour frapper son lecteur, à signaler des épisodes piquants de la vie de tel ou tel individu, en les montant en épingle au moyen du paradoxe, comme dans le cas du Macédonien Persée, qui jette son trésor dans les flots pour le préserver de l'ennemi ${ }^{28}$, ou encore de l' exagération, comme pour le roitelet Épulon, hissé sur son cheval alors qu'il est tout à fait ivre $^{29}$.

\section{Obstacle compositionnel : historiographies chronologique et thématique}

17 Une dichotomie simple pour analyser l'historiographie et la biographie antiques consiste à distinguer une composition chronologique et une composition thématique. La première tendrait à énumérer, pour chaque année, les événements advenus (Tite-Live, pour l'historiographie; Cornélius Népos, avec des nuances, pour la biographie); la seconde envisage de grands ensembles suivant un autre ordre que la succession chronologique (Appien, pour l'historiographie, suit une démarche géographique ; Suétone, pour la biographie, opère souvent des groupements fondés sur les vices et les vertus de ses objets d'étude).

\section{Un cadre mal adapté à la biographie}

\section{Contraintes liées à la composition chronologique}

Il est certain que la première méthode est la moins propice à la biographie, puisqu'elle fragmente la vie des héros en diverses années, et ne permet guère d'appréhender la figure d'un individu dans son ensemble ${ }^{30}$.

19 Or l'œuvre de Florus, si elle n'étudie certes pas les événements année par année, respecte du moins, dans l'ensemble, l'ordre chronologique : les éléments biographiques caractérisant un individu, comme sa naissance ou sa mort, ne pourraient donc, la plupart du temps, être évoqués que dans le cadre d'analepses ou de prolepses rompant 
le cours du récit, au risque de nuire à l'intelligence de celui-ci; si de telles distorsions narratologiques se conçoivent dans les récits de quelque ampleur ${ }^{31}$, elles sont d'un maniement particulièrement délicat dans le cadre d'une écriture annalistique brève.

\section{Contraintes liées à la composition thématique}

Mais il y a pire. Bien que l'œuvre de Florus comporte aussi des éléments thématiques, ceux-ci sont eux-mêmes fort peu favorables à l'écriture d'un texte de nature biographique : il existe, d'abord, une bipartition principale entre les guerres externes (livre I) et les guerres internes (livre II) ; en outre, au sein de chacun de ces livres, les chapitres s'organisent autour d'un événement principal (une guerre ou une sédition) ${ }^{32}$, sans compter le primat accordé à la géographie sur la chronologie ${ }^{33}$. Tout cela nuit à une vision cohérente globale: Pompée, par exemple, est pour ainsi dire écartelé en trois endroits : il est évoqué en I, 40-41 pour son action énergique contre Mithridate et contre les pirates (guerres extérieures), puis en II, 10-11 pour les victoires remportées contre Drusus et contre Sertorius, avant d'être l'objet d'une plus grande attention pour la guerre qui l'opposa à César (II, 13) ${ }^{34}$. Dès lors, c'est une figure kaléidoscopique qui se dégage, et ce d'autant plus que Florus se dispense très souvent de faire le lien entre ces moments épars : présupposant une connaissance assez large des événements chez ses lecteurs ${ }^{35}$, il ne juge pas bon, par exemple, de nommer Camille, encore anonyme quand il est question de sa générosité à Faléries (I, 6,5); son nom n'apparaît que lorsqu'il chassera les Gaulois d'Italie (I, 7, 19). Dans ces circonstances, il est impossible d'établir un continuum biographique pour le lecteur de Florus.

\section{Des accommodements avec l'histoire pour assouplir un cadre trop rigide}

21 C'est peut-être là que la difficulté de Florus est la plus grande. Il s'efforce toutefois de la surmonter, en n'hésitant pas à modifier les données chronologiques et historiques.

Quand un personnage a une biographie trop pauvre en effet, l'historien met parfois sur son compte des éléments concernant un tiers: c'est ainsi qu'il procède avec les Gracques, en attribuant à Caius des actes accomplis par Tibérius pour conserver un équilibre entre les deux personnages ${ }^{36}$. Cette subordination délibérée de la biographie au style et à la composition explique d'ailleurs bien des anachronismes reprochés à l'épitomateur ${ }^{37}$.

\section{Contrainte idéologique : rôles de la fortuna et du populus Romanus}

23 La préface de Florus est sans doute la partie de son œuvre la mieux connue, parce qu'elle présente deux réflexions d'ordre idéologique qui n'ont pas le mérite de l'originalité, mais qui ont du moins celui de donner une grande cohérence à l'ensemble du récit: premièrement, l'histoire de Rome a été régie par les deux forces fondamentales que sont la fortuna et la uirtus; deuxièmement, la croissance de Rome peut s'assimiler à celle que connaît un individu de sa naissance à sa mort. 


\section{Des individus relégués au second plan...}

\section{Rôle de la fortuna} de l'histoire qui a déjà été bien analysée ${ }^{39}$ : ici, la Fortuna n'est pas un hasard aveugle, mais une forme de providence qui gouverne le monde - ou, du moins, le peuple romain ${ }^{40}$. À l'extrême, elle réduit les individus à des pantins, dont il est inutile de présenter les motivations : cette surdétermination ne laisse plus qu'une part modique aux facteurs biographiques. S'il serait exagéré de prétendre que Florus néglige totalement la psychologie, il demeure cependant exact que ses personnages paraissent constamment les jouets de la fortuna, si bien que les racines de leurs actions se trouvent rarement dans leur biographie ${ }^{41}$. Les Gaulois Sénons, par exemple, envahissent Rome siue inuidia deum siue fato, c'est-à-dire à cause de la « jalousie des dieux » ou de la « fatalité », et non en raison des desseins de leurs chefs (I, 7, 1); si Antiochus déclenche une guerre contre Rome, c'est quodam casu, quasi de industria sic adgubernante fortuna, "par une sorte de hasard» ou "comme si la fortune était aux commandes » (I, 23, 1); du côté romain, Scipion l'Africain devra son succès au fatum (I, 22, 37), et d'autres exemples pourraient être cités ${ }^{42}$.

\section{Rôle du populus Romanus}

Florus annonce en outre qu'il racontera les progrès du peuple romain comme si celui-ci était un organisme humain : son enfance, sa jeunesse, sa fière virilité et sa vieillesse constituent en effet les quatre "âges» de Rome ${ }^{43}$. Peu importent, dans notre perspective, les racines profondes de cette conception ${ }^{44}:$ il demeure en tout cas que, pour Florus, ce sont les peuples, non les individus, qui font l'histoire ${ }^{45}$. Le résultat est que la seule entité que l'écrivain traite d'une façon ouvertement biographique est le peuple romain lui-même ${ }^{46}$, unique agent véritable du développement de l'Empire ${ }^{47}$. Le populus Romanus devenant lui-même le héros et le modèle de l'ouvrage, la fonction d'exemplarité habituellement dévolue aux grands hommes, qui justifie l'écriture biographique dans l'Antiquité classique, disparaît, et c'est une raison de moins de mettre en valeur telle ou telle individualité ${ }^{48}$.

Dans ces conditions, le nom même des chefs romains est parfois passé sous silence ${ }^{49}$, et leur destin individuel - leur mort, par exemple - presque toujours laissé de côté ${ }^{50}$. Probablement y a-t-il là un héritage de la pratique historiographique de Caton l'Ancien, si populaire sous les Antonins ${ }^{51}$, lui aussi adepte de la breuitas ${ }^{52}$ et plaçant la collectivité romaine tellement au-dessus des individualités, même brillantes, qu'il rapportait seulement les faits, en taisant les noms des protagonistes ${ }^{53}$.

Le résultat dans l'écriture est le suivant : le peuple romain est l'agent des événements, et les magistrats du peuple roi ne sont mentionnés qu'à travers un ablatif absolu (duce, consulibus...) qui les relègue pratiquement au rôle de simples spectateurs : Sic Pompeio 
duce populus Romanus totam, qua latissima est, Asiam peruagatus («Ainsi, sous la conduite de Pompée, le peuple romain parcourut l'Asie à l'endroit où elle est la plus large » ${ }^{54} \ldots$ Une autre manifestation de cette habitude est le fréquent recours à la voix passive : même César, si énergique dans sa conquête des Gaules, est ainsi laissé dans l'ombre, en n'étant le sujet d'aucun verbe, à une exception près ${ }^{55}$.

\section{... dont l'émergence soudaine remplit une fonction idéologique profonde}

\section{Montée en puissance du biographique chez les chefs étrangers}

Cette pratique de Florus favorise cependant une montée en puissance de la biographie des chefs étrangers : un parallèle est régulièrement dressé entre le peuple romain et un chef ennemi. La dissymétrie, qui résulte d'institutions différentes (République vs monarchie), valorise pour ainsi dire mécaniquement le chef ennemi comme individualité. Hannibal constitue à cet égard un bon exemple: le peuple romain se confronte au Punique, qui incarne l'ensemble de sa nation, au sein de laquelle les autres personnalités sont reléguées à l'arrière-plan ${ }^{56}$. Voilà qui rejoindrait, d'une certaine façon, des vers attribués à un certain Florus, qui ne fait probablement qu'un avec notre historien ${ }^{57}$, et qui met en valeur les reges étrangers, dotés d'un aspect fascinant, par opposition aux consules romains, plus falots :

Consules fiunt quotannis et noui proconsules:/ solus aut rex aut poeta non quotannis nascitur

Des consuls, on en crée chaque année, et de nouveaux proconsuls ; / seul le roi ou le poète ne naît pas chaque année ${ }^{58}$.

\section{Émergence d'individualités romaines dans le second livre}

De surcroît, du côté romain, les figures individuelles commencent à émerger dans le second livre, au détriment du populus Romanus ${ }^{59}$ : Tibérius Gracchus (II, 2), par exemple, reçoit beaucoup d'informations de type biographique : sa naissance $(\$ 1)$, sa carrière $(\$ 2-6)$ et sa mort (\$7) sont successivement rapportées, informations auxquelles s'ajoutent des réflexions politiques $(\$ 2)$ et psychologiques $(\$ 3)$ sur les motivations de ses actions, qu'il n'était pas nécessaire d'évoquer du strict point de vue du populus Romanus.

Cette façon de souligner l'importance d'individus, en même temps qu'elle a une valeur littéraire certaine, et qu'elle s'explique par le rôle croissant que jouent les individus eux-mêmes dans les institutions et dans l'histoire romaine ${ }^{60}$, a sans doute aussi pour but d'illustrer la dégénérescence de l'État. Parallèlement, en effet, à des déclarations sur la déliquescence du populus Romanus en tant qu'organisme ${ }^{61}$, la multiplication des individus décrits d'une façon biographique vient souligner le naufrage moral dans lequel Rome est plongé. Cette orgie biographique a donc sa raison d'être d'un point de vue compositionnel: le foisonnement des destinées individuelles crée une bigarrure, une instabilité et un désordre qui brisent le bel ordonnancement du premier livre, dans lequel les individus disparaissaient derrière le populus Romanus: l'unité disparaît derrière la fragmentation, la beauté de la collectivité derrière la médiocrité des carrières personnelles. 


\section{Conclusion}

Chez Florus, trois facteurs réduisent nécessairement la part du biographique: la recherche permanente de la breuitas, sur un plan stylistique; la double méthode annalistique et thématique, sur un plan historiographique; la place accordée à la fortuna et au populus Romanus, sur un plan idéologique. À plusieurs reprises cependant, l'historien se dégage de contraintes trop étouffantes et parvient à donner vie à certains héros, en redoublant de virtuosité (biographies miniatures), en bouleversant les données chronologiques et historiques ("étoffement» de certains personnages) ou surtout en faisant de l'écriture biographique un élément signifiant sur le fond, puisqu'elle finit par traduire la dégénérescence qui frappe la République romaine, d'où disparaît progressivement, dans le fracas des guerres civiles, tout sentiment de relever d'un destin commun.

\section{Textes anciens}

Florus, Cuvres, éd. P. Jal, CUF. Série latine 163 et 188, Paris, 1967, 2 vol.

L. Annaei Flori Epitomae libri II, et P. Annii Flori Fragmentum de Vergilio oratore an poeta, éd.

O. Rossbach, Bibliotheca scriptorum Graecorum et Romanorum Teubneriana, Leipzig, 1896.

\section{Textes modernes}

ALBA V. 1953, La concepción historiografíca de L. Anneo Floro, Madrid.

ALONSO-NUÑEZ J.M. 1983, Die politische und soziale Ideologie des Geschichtsschreibers Florus, Bonn.

ALONSO-NUÑEZ J.M. 1986, « Les conceptions politiques de Florus », LEC 54, p. 178-180.

BALDWIN B. 1988, « Four Problems with Florus », Latomus 47, p. 134-142.

BAYET J. 1965, Littérature latine, nouv. éd. mise à jour avec la collab. de L. Nougaret, Coll. U, Paris.

BERTHAUT H., GEORGIN C. 1923, Histoire illustrée de la littérature latine, Paris.

BESSONE L. 1979, « Ideologia e datazione della Epitoma di Floro », Giornale filologico Ferrarese 2, p. 33-57.

BESSONE L. 1992-1993, « Floro: anacronismi per omissione », AIV 151, p. 391-410.

BESSONE L. 1993, «Floro: un retore storico e poeta », ANRW II, 34, 1, p. 80-117.

BESSONE L. 1994a, « Alla riscoperta di Floro », A\&R 39, p. 77-84.

BESSONE L. 1994b, « Fra storiografia e biografia: Floro e l'età regia », ACD 30, p. 223-230. 
BESSONE L. 1996, La storia epitomata: introduzione a Floro, Problemi e ricerche di storia antica 19, Rome.

BRIZZI G. 1984, «Imitari cœpit Annibalem (Flor. I, XXII, 55): apporti catoniani alla concezione storiografica di Floro?", Latomus 43, p. 424-431.

CAVAGGIONI F. 2004-2005, « Il Saturnino di Floro: elementi di continuità, omissioni e incongruenze nel rittrato di un seditiosus ", ACD 40-41, p. 325-337.

CHAMoux F. 1997, « La biographie dans la Bibliothèque historique de Diodore de Sicile ", in J.A. Sánchez Marín, J.L. Tuero, C. López Rodríguez (éds), Historiografía y biografía: actas del coloquio internacional sobre historiografía y biografía (de la Antigüedad al Renacimiento), Granada, 21-23 septiembre de 1992, Madrid, p. 57-65.

DELLA CORTE F. 1949, Catone censore, la vita e la fortuna, Turin.

DEN BOER W. 1965, « Florus und die römische Geschichte », Mnemosyne 18, p. 365-387.

DESCHAMPS L. 1989, « Subtil Florus !... ou le 'poète-roi' », REA 91, p. 89-93.

EDWARDS M.J. 1997, « Biography and the Biographic », in M.J. Edwards, S.C.R. Swain (éds), Portraits: Biographical Representation in the Greek and Latin Literature of the Roman Empire, Oxford, p. 227-234.

GAILLARD J. 1992, Approche de la littérature latine: des origines à Apulée, 128. Lettres 7, Paris.

GARZETTI A. 1964, «Floro e l'età Adrianea », Athenaeum 42, p. 136-156.

GASCOU J. 1984, Suétone historien, Bibliothèque des Écoles françaises d'Athènes et de Rome 255, Rome.

GENTILI B., CERRI G. 1988, History and Biography in Ancient Thought, London Studies in Classical Philology 20, Amsterdam [trad. de : Storia e biografia nel pensiero antico, Rome, 1983].

GIUA M.A. 1990, « Aspetti della biografia latina del primo impero », RSI 102, p. 535-559.

HAVAS L. 1988, «Zum aussenpolitischen Hintergrund der Entstehung der Epitome des Florus », $A C D$ 24, p. 57-60.

HINOJO A.G. 1983, «Juicio de los historiadores imperiales sobre los Gracos », Helmantica 34, p. 293-308.

HOSE M. 1994, Erneuerung der Vergangenheit: die Historiker im Imperium Romanum von Florus bis Cassius Dio, Beiträge zur Altertumskunde 45, Stuttgart - Leipzig.

JAL P. 1965, « Nature et signification politique de l'ouvrage de Florus », REL 43, p. 358-383.

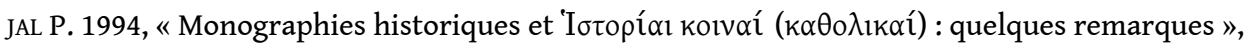
REL 72, p. 56-78.

JAL P. 1997, « Historiographie annalistique et historiographie thématique dans l'Antiquité classique : quelques remarques », REL 75, p. 27-37.

JAL P. 1999, C.R. de L. Bessone, La storia epitomata: introduzione a Floro, Problemi e ricerche di storia antica 19, Rome, 1996, Latomus 58, p. 901-903.

LEUZE O. 1911, « Die Darstellung des 1. punischen Kriegs bei Florus », Philologus 70, p. 549-560.

LÓPEZ MELERO R. 1988, « Viriatus Hispaniæ Romulus », in J.J. Sayas Abengoechea, A. Fernández Vega, S. Ripoll López (éds), Homenaje al profesor Eduardo Ripoll Perelló, Espacio, tiempo y forma. Serie II, Historia antigua, Madrid, p. 247-261. 
MACÉ A. 1900, Essai sur Suétone, Bibliothèque des Écoles françaises d'Athènes et de Rome 82, Paris.

MAZZA M. 1965, « Sulla tematica della storiografia romana di epoca sillana: il fr. 1-2 Peter di Sempronius Asellio », SicGymn 18, p. 144-163.

MOMIGLIANO A. 1971, The Development of Greek Biography: Four Lectures, Cambridge (Mass.).

MONCEAUX P. 1894, Les Africains : étude sur la littérature latine d'Afrique, Nouvelle bibliothèque littéraire, Paris.

MORENO I. 1991-1992, « El recurso enumerativo en los breviarios latinos », Veleia 8-9, p. 423-437.

NEUHAUSEN K.A. 1994, « Der überhörte ‘Schwanengesang' der augusteischen Literatur: eine Rekonstruktion der Originalfassung (um $15 \mathrm{n}$. Chr.) des bischer dem 2. Jh. Zugeordneten Geschichtswerkes des Florus ", ACD 30, p. 149-207.

NORDH A. 1951, « Virtus and Fortuna in Florus », Eranos 49, p. 111-128.

PELLING C. 1997, «Biographical History? Cassius Dio on the Early Principate », in M.J. Edwards, S.C.R. Swain (éds), Portraits: Biographical Representation in the Greek and Latin Literature of the Roman Empire, Oxford, p. 117-144.

PICCIRILLI L. 2000, « Diodoro tra biografia e storia », SIFC 18, p. 112-118.

PIERRON A. 1882, Histoire de la littérature romaine, $11^{\mathrm{e}}$ éd. augmentée, Paris.

SCHANZ M. 1922, Geschichte der römischen Literatur, bis zum Gesetzgebungswerk des Kaisers Justinian. III, Die Zeit von Hadrian 117 bis auf Constantin 324, dritte neubearbeitete Aufl., Handbuch der Altertumswissenschaft 8, Munich.

SWAIN S.C.R. 1997, «Biography and Biographic in the Literature of the Roman Empire ", in M.J. Edwards, S.C.R. Swain (éds), Portraits: Biographical Representation in the Greek and Latin Literature of the Roman Empire, Oxford, p. 1-37.

VENERONI B. 1974, «Quatenus, qua ratione res politicas et sociales Florus tractaverit 》, Aevum 48, p. 345-348.

\section{NOTES}

1. Déterminer le titre de l'œuvre de Florus a mobilisé une grande partie des efforts de la recherche; la proposition de Jal (éd.) 1967, I, p. XXI-XXIII (tabella, «tableau », encore défendue par Jal 1999, p. 902) semble douteuse (Veneroni 1974, p. 345, n. 1 ; Baldwin 1988, p. 138-139; Bessone 1993, p. 84). Par convention, nous parlerons d'Epitome, jugeant la proposition de Bessone 1996, p. 17 («la prétendue Epitome de Tite-Live»), plus fondée scientifiquement, mais moins commode pratiquement. - Pour l'établissement et la traduction du texte de Florus, nous suivons l'édition de P. Jal citée au début de cette note.

2. Pierron 1882, p. 585 : «Il n'est pas douteux qu'on ne doive placer l'auteur au premier rang des rhéteurs et des déclamateurs romains. Mais c'est là un triste éloge, si l'on veut voir dans Florus un historien »; Schanz 1922, p. 67 : «Sein Buch ist in der Tat kein Geschichtswerk [...] sondern ein Panegyrikus »; p. 70 : «Florus darf nicht als Historiker beurteilt werden »; Berthaut, Georgin 1923, p. 436 : «L'auteur <se> livre à un panégyrique outrancier du peuple romain et accumule les erreurs, les contradictions, les oublis »; Bayet 1965, p. 419 : «Omissions et erreurs n'y sont pas rares [...] un style à effets, plein d'exagérations, d'expressions poétiques, d'exclamations inutiles»; Gaillard 1992, p. 116 : «... une prose si préoccupée d'ornements rhétoriques qu'elle exhibe plus de figures que d'indications historiques ». 
3. Bessone 1994a, p. 81, n. 22.

4. La date précise à laquelle Florus écrit n'est pas connue, mais il s'agit sans doute du début du II s. apr. J.-C. (peu défendable paraît être la datation augustéenne [Neuhausen 1994: original de l'époque d'Auguste, ayant subi des modifications ultérieures], et l'hypothèse de Trajan a été abandonnée; sous Hadrien : Monceaux 1894, p.198-199; Schanz 1922, p. 70 ; Garzetti 1964 ; Jal 1965 ; Jal [éd.] 1967, I, p. XLI-XLIII, puis CIV-CXI ; Veneroni 1974, p. 348 ; Brizzi 1984 ; Hose 1994, p. 56-61; sous Antonin : Havas 1988 ; Bessone 1993, p. 97-102, suivi désormais par Jal 1999, p. 903), moment où la biographie connaît un incontestable engouement (Momigliano 1971, p. 99-100, et, dans l'ensemble, Swain 1997, praes. p. 22-23).

5. Hose 1994, p. 80.

6. En faveur d'une influence de cet ordre, cf. Jal (éd.) 1967, I, p. LIV-LVII, suivi par Bessone 1993, p. 112 ; Hose 1994, p. 74-76, estime même que l'ouvrage tout entier est une biographie du peuple romain écrite à la manière de Suétone, mais n'indique pas d'influence directe de Suétone sur Florus; contra Macé 1900, p. 104-105, pour des motifs futiles (animosité personnelle entre les deux écrivains, que tout séparait). - En ce qui concerne le contenu, on pense par ailleurs que Florus avait lu plusieurs ouvrages biographiques pour composer son Epitome, notamment Hygin (Monceaux 1894, p. 200 ; Bessone 1994b, p. 229-230) et Suétone (Gascou 1984 remarque que si Florus, utilisant des sources anti-césariennes comme Tite-Live ou Lucain, est généralement éloigné de Suétone, il a pu lui faire des emprunts ponctuels, non sans déformer sa pensée d'ailleurs : p. 36-37, à propos de la fuite de César à la nage ; p. 155 et n. 223, à propos du ueni, uidi, uici).

7. Le cas de Velleius Paterculus est un peu différent, dans la mesure où, tout à son travail de panégyriste, il accorde une très large place à César, Auguste et Tibère, et réalise donc de facto une œuvre de biographe (cf. Jal 1994, p. 60 ; Pelling 1997, p. 122).

8. Bessone 1996, p. 135-137.

9. Jal (éd.) 1967, I, p. XLIX-LI.

10. Sur le manque de place laissée à la biographie, et les inconvénients qui en résultent, dans une histoire à prétention universelle du type de celle de Florus, cf. Jal 1994, p. 60.

11. Pour Diodore, cf. Chamoux 1997 ; pour Dion Cassius, cf. la fine analyse de Pelling 1997, praes. p. 117-125, élaborant le concept de «biostructure»: sans être strictement biographique (de nombreux détails concernant l'individu sont laissés dans l'ombre), l'approche de Dion Cassius, pour la période qui concerne le début du Principat, met au centre un homme (empereur ou Grand de l'État) autour duquel s'articulent tous les événements du règne envisagé.

12. À ce moment de notre exposé, une clarification d'ordre lexical s'impose, qui distingue biographie et biographique. Il est clair que Florus n'a pas écrit de biographie, conçue comme un genre littéraire en soi, sauf à considérer qu'il est le biographe du peuple romain (cf. supra, n. 6). Mais, de la même façon que le tragique ne se réduit pas à la tragédie, le biographique ne se réduit pas à la biographie (analogie que nous empruntons à Edwards 1997, p. 227). Nous préférons donc parler, dans un cadre plus vaste, de biographique, pour lequel nous retenons la définition de Swain 1997, p. 1-2 : «Biographical texts are texts which furnish detailed accounts of individuals' lives. They may be complete, from birth to death, or sectional and partial». Cette définition plus large permet d'éviter d'opposer trop strictement et artificiellement biographie et historiographie, qui, dans l'Antiquité, peuvent cohabiter et interagir, comme l'a bien montré l'analyse de Gentili, Cerri 1988, praes. p. 61-66, notamment au sujet de Pol. 10, 21 (cf. aussi Chamoux 1997, p. 5 ; à propos de Florus et des ouvrages historiques brefs en général, cf. également les remarques de Bessone 1994b, p. 230).

Pour le dire d'un mot, nous considérons que tout passage qui évoque la vie d'un individu n'est pas biographique per se, mais que le biographique n'est pas non plus systématiquement circonscrit dans des Vies ou dans des excursus bien délimités au sein d'un récit plus large. Ainsi, nous considérerons qu'un passage évoquant la vie d'un individu est de nature historiographique s'il est 
utilisé comme pure cause des événements mis en récit (comme c'est généralement le cas chez Florus), mais qu'il est de nature biographique s'il est envisagé en lui-même, pour donner des précisions sur tel ou tel individu.

Pour illustrer notre position à l'aide d'un cas caricatural, disons que, dans la phrase «Caligula, atteint d'épilepsie, fut pris pour un fou ", l'élément souligné n'est pas de nature biographique, puisqu'il opère un lien causal direct entre l'information et l'événement mis en récit, alors qu'il l'est dans la phrase "Caligula, atteint d'épilepsie, succéda à Tibère », puisqu'on ne peut opérer de relation de cause à effet entre l'information et l'événement. Évidemment, dans bien des situations, ce critère ne peut s'appliquer de façon très claire : nombreuses sont les zones grises.

13. Cavaggioni 2004-2005, p. 326-327, a relevé ce procédé au sujet du personnage de Saturninus, dont seul le second tribunat retient l'attention de Florus; il est vrai, d'autre part, que, parmi les historiens anciens, ce trait n'est pas propre à Florus en ce qui concerne Saturninus.

14. Chamoux 1997, p. 65 . Il convient toutefois, à ce propos, d'être prudent: les Anciens n'attendaient pas d'un historien, ni même d'un biographe, qu'il accorde une grande importance à la psychologie personnelle de ses personnages (cf. le salutaire caueat de Pelling 1997, p. 138).

15. Hinojo 1983, p. 297, l'a relevé à propos des Gracques.

16. Flor., I, 42, 4-5.

17. Sur le dédain pour l'analyse étiologique comme caractéristique de l'écriture annalistique, cf. Sempronius Asellio (Chassignet 1, 2-3) et le commentaire de Mazza 1965, praes. p. 151-159.

18. Cf. Moreno 1991-1992, p. 430-431.

19. Flor., 1, 39, 5-6.

20. Flor., 2, 6, 13 : Adgressique singulos populos Cato discutit Etruscos, Gabinius Marsos, Carbo Lucanos, Sulla Samnites («Attaquant les peuples séparément, Caton écrase les Étrusques, Gabinius, les Marses, Sylla, les Samnites »).

21. Bessone 1996, p. 45-46, a formulé une remarque de cet ordre au sujet des rois, mais on pourrait l'étendre à presque tous les personnages de l'Epitome.

22. Flor., 2, 21, 2.

23. Flor., 1, 33, 15.

24. Comme le note López Melero 1988, p. 248.

25. Telle était, par ex., l'opinion de Juste Lipse. L'hypothèse d'une origine africaine (Bessone 1996, p. 152-153) est aujourd'hui dominante, mais il est de toute façon acquis que Florus a fait en Hispanie un séjour plaisant (Flor., V. O. A. P., 1, 6;2, 6-8). L'éloge de l'Hispanie pourrait aussi se comprendre par référence à Hadrien, originaire de cette province (Gel., 16, 13, 4 ; cf. Garzetti 1964, p. 156), ou, plus globalement, au rôle dévolu à cette région dans la politique impériale depuis Trajan (Hose 1994, p. 134-136).

26. Flor., 2, 8, 8 : Nec abnuit ille de stipendiario Thrace miles, de milite desertor, inde latro, deinde in honorem uirium gladiator ( Et celui-ci ne refusa pas, lui qui, de tributaire thrace, était devenu soldat, de soldat déserteur, puis brigand et ensuite - honneur dû à sa force - gladiateur »).

27. Plut., Alex., 1, 2.

28. Flor., 1, 28, 6: Cujus tanta trepidatio fuit, ut pecuniam omnem in mare iusserit mergi ne periret, classem cremari ne incenderetur ( Si grande fut sa frayeur, qu'il ordonna de jeter tout le trésor à la mer, pour qu'il ne disparût pas, et de brûler la flotte, de peur qu'elle ne fût incendiée »).

29. Flor., 1, 26, 3 : Ipse rex Aepulo equo inpositus, cum subinde crapula et capitis errore lapsaret, captum sese uix et aegre, postquam expergefactus est, didicit («Le roi Épulon lui-même, alors qu'il glissait du cheval sur lequel on l'avait hissé à cause du trouble que lui causait son ivresse, ne comprit qu'à grand-peine, à son réveil, qu'il avait été fait prisonnier »).

30. Sur tous ces points, cf. la synthèse de Jal 1997 pour l'historiographie; les remarques de Momigliano 1971, p. 18-20, pour la biographie.

31. À propos de Philippe II de Macédoine chez Diodore de Sicile, cf. Chamoux 1997, p. 62. 
32. Sur la coexistence, dans l'Epitome, des deux types d'historiographies (chronologique et thématique), cf. Jal 1997, p. 35-36.

33. En lisant l'étude de Leuze 1911, praes. p.511-512, consacrée au récit de la première guerre punique chez Florus, on voit bien comment l'action romaine se distribue par grandes zones géographiques, au lieu de se répartir, comme on aurait pu l'attendre, par généraux.

34. Les personnages de Sylla (1, 40, 9-12: guerre contre Mithridate ;, 9 : guerre contre Marius), Marius (1, 38 : contre les Teutons et les Cimbres ; 2,9 : contre Sylla) et César (1, $45:$ contre les Gaulois ; 2,13 : contre Pompée) pourraient d'ailleurs appeler les mêmes remarques.

35. Bessone 1979, p. 36 et n. 16 ; Hose 1994, p. 65. Aussi nous paraît-il erroné d'y voir, comme Den Boer 1965, p. 369 et 382 , un ouvrage scolaire.

36. Le point dont il est ici question est celui de l'utilisation de l'héritage du roi Attale; cf. Bessone 1996, p. 109, n. 3 : «bilanciare il poco spazio concesso a Gaio ridotto a fotocopia del fratello » (hypothèse préférable selon nous à celle de la simple erreur défendue par Jal [éd.] 1967, II, p. 14, n. 1, qui reste cependant défendable, eu égard à la confusion généralisée existant, dans l'Antiquité, entre Caius et Tibérius Gracchus [nous remercions M. G. Salamon pour les remarques qu'il nous a faites sur ce point]).

37. Problème étudié par Bessone 1992-1993.

38. Flor., proem., 2.

39. Hose 1994, p. 96-103.

40. Providence universelle (i.e. « polybienne ») : Hose 1994, p. 97-99; fortuna guidant seulement le peuple romain : Alonso-Nuñez 1983, p. 9.

41. À cet égard, cf. les remarques ponctuelles de Alba 1953, p. 48-49, surtout à propos de César et de Pompée, et de la mystérieuse protection dont ils bénéficient de la part de la fortuna; Hose 1994, p. 108: «... der menschliche Faktor, die psychologischen Triebkräfte, im Hintergrund bleiben ». On prendra garde, de toute façon, à ne pas surestimer le rôle de la psychologie dans l'historiographie et même dans la biographie des Anciens.

42. Flor., 1, 31, 12: hoc scilicet fato, à propos des succès de Scipion Émilien ; 2, 9, 8 : dans un premier temps, Marius échappe à la mort seulement parce que «la fortune l'a réservé pour un autre combat » (fortuna alteri bello reseruauit) ; une phrase du même type sert de commentaire au sort d'abord heureux de Sextus Pompée en Celtibérie $(2,13,87)$. Cf. encore les remarques de Den Boer 1965, p. 385-386.

43. Flor., proem., 4-8.

44. Alba 1953, p. 29-31, met en avant une influence stoïcienne, mais ses arguments sont plutôt ténus.

45. Cf. e. g. Alonso Nuñez 1986, p. 179.

46. Hose 1994, p. 70, intitule d'ailleurs un des chapitres de son essai : «Florus als Biograph des populus Romanus ".

47. Cf. déjà Monceaux 1894, p. 205 : «S'il esquisse un portrait d'homme ..., c'est en quelques mots »; Jal (éd.) 1967, I, p. XXXIX-XL.

48. Sur la justification de la biographie comme source d'édification pour les lecteurs, cf. e.g. Pol., 10, 21, 3-4 ; D.S., 10, 12 (éd. Odlfather; sur ce passage, les réflexions de Piccirilli 2000 ont un intérêt pour l'écriture biographique dans son ensemble); Plut., Tim., praef., 5 ; en général Giua 1990, p. 539-540.

49. Rossbach (éd.) 1896, p. LI : « Omnes res a populo Romano ipso gestas esse fingit, ducum vero nomina saepe omittit ».

50. Hose 1994, p. 90 : «Die Konzeption des Florus, den populus Romanus zum Helden der ersten Werkhälfte $\mathrm{zu}$ machen, führt $\mathrm{zu}$ einer Reduktion der Rolle römischer Feldherren, die als Gestalten ein entsprechend geringes Gewicht erhalten. So ist wenig Raum für die Darstellung der clari ducum exitus». 
51. Pour Hadrien, cf. SHA, Hadr., 16, 6 : Ciceroni Catonem ... praetulit ( « <Hadrien> plaçait Caton audessus de Cicéron ») ; pour ses successeurs, Della Corte 1949, p. 162-163.

52. Sall., Hist., 1, 4 (ed. Maurenbrecher) : Romani generis disertissimus <summa ?> paucis absoluit ; Nep., Cato , 3, 4 : Haec omnia capitulatim sunt dicta (mais le sens de capitulatim est discuté) ; Bessone 1993, p. 113.

53. Nep., Cat., 3, 4 : Duces non nominauit, sed sine nominibus res notauit (« Au lieu de nommer les chefs, il a consigné les événements sans donner de noms »). Sur ce point commun entre Caton et Florus, et l'influence qu'il implique de la part du premier sur le second, cf. Rossbach (éd.) 1896, p. LI-LII ; Bessone 1994a, p. 80. Ce type d'influence avait, peut-être, déjà joué sur Tacite (Giua 1990, p. 557). Nordh 1951, p. 111, a relevé que l'antilogie Fortuna / uirtus était déjà présente chez Caton ; Brizzi 1984 discerne lui aussi une influence de Caton sur Florus, en se plaçant dans une perspective un peu différente.

54. Flor., 1, 40, 31 ; cf. encore 1, 8, 1; 1, 9, 1; 1, 10, 2... Schanz 1922, p. 67.

55. Flor., 1, 45, 3-8.

56. Flor., 1, 22, notamment $\S 55$ (sur ce passage, on se reportera à l'étude détaillée menée par Brizzi 1984).

57. Cf. dans ce sens Baldwin 1988, p. 314-317.

58. Flor., Poemata, X. Analysant ce distique, Deschamps 1989, p.93, souligne la prééminence qu'acquiert, aux yeux de Florus, la figure du roi (au point qu'il veut s'y réfugier après l'humiliation subie aux Jeux capitolins).

59. Cf. Hose 1994, p. 73-74.

60. L'émergence des figures individuelles dans les livres de Dion Cassius consacrés au début du Principat a ainsi été mise en corrélation avec le développement du pouvoir personnel par Pelling 1997 , p. 121 ; cf. aussi D.C., 53, 19, 3-4.

61. Flor., 1, 47, 3-13 ; 2, 3, 6 ; 2, 5, 3 ; cf. Jal (éd.) 1967, I, p. XL-XLI.

\section{RÉSUMÉS}

L'article interroge la place que peut prendre dans le genre de l'Epitome cultivé par Florus la biographie d'agents individuels en montrant comment Florus y négocie avec trois contraintes : la brièveté imposée par le genre, la coexistence d'une méthode chronologique et d'une méthode thématique, et la vision particulière du monde qui sous-tend l'œuvre. On montre que ces contraintes n'empêchent pas Florus de se dégager de contraintes trop étouffantes et de donner vie à certains héros, en redoublant de virtuosité, en bouleversant les données chronologiques et historiques ou surtout en faisant de l'écriture biographique un élément signifiant sur le fond, puisqu'elle finit par traduire la dégénérescence qui frappe la République romaine, d'où disparaît progressivement tout sentiment de relever d'un destin commun. 
INDEX

Mots-clés : biographie, breuitas, historiographie romaine, individu, peuple romain, portrait oeuvrecitee Histoire Auguste

nomsmotscles Appien, Caton l'Ancien, Diodore de Sicile, Dion Cassius, Florus, Plutarque, Polybe, Salluste, Sempronius Asellio, Suétone

Index géographique : Faléries, Gaule, Italie

Keywords : biography, brevitas, Roman historiography, individual, Roman people, portrait

\section{AUTEURS}

GUILLAUME FLAMERIE DE LACHAPELLE

Université de Bordeaux 3, UMR 5607 Ausonius

Maître de conférences de langue et littérature latines 